\title{
Evaluation and Analysis of Uncertainty Measurement of The Sound Level Meter Calibration by Coupler Method
}

\author{
Bondan Dwisetyo and Denny Hermawanto \\ Badan Standardisasi Nasional, Kompleks PUSPIPTEK Gedung 420, Serpong, Tangerang Selatan 15314
}

\begin{abstract}
The calibration of sound level meter (SLM) using a coupler method has been introduced. This method utilizes a multifunction acoustic calibrator as a portable laboratory instrument. By the reason of its convenient application, it is appropriate to be realized by the secondary calibration laboratory. To complete the requirement of calibration result provided by ISO 17025, an analysis of the uncertainty measurement related to this calibration is strongly needed. Therefore, this work aims to provide an evaluation and analysis of some elements that influence to the coupler method of SLM calibration using the statistical approximation to determine the major parameters such as standard uncertainty, combine uncertainty, degree of freedom, and expanded uncertainty in accordance with The Guide to the expressions of Uncertainty in Measurement (GUM). Moreover, a case study related to this instrument calibration also discussed along this paper. From the result, the uncertainty budgets that contribute to the SLM calibration has been obtained, and generally, it is found that the standard instrument has the highest contribution to the determination of the expanded uncertainty. For the whole results, the obtained uncertainty values are still acceptable according to the acceptance limit values that required by IEC $61672-1$
\end{abstract}

Keywords: calibration of sound level meter; coupler method; GUM; uncertainty measurement.

*Corresponding author: bondan@bsn.go.id

Article history: Received 2 May 2020, Accepted 25 August 2020, Published January 2021 http://dx.doi.org/10.12962/j24604682.v17i1.6988

2460-4682 @DDepartemen Fisika, FSAD-ITS

\section{INTRODUCTION}

The calibration of the acoustical instruments such as a microphone, a sound calibrator, and a sound level meter, has become the trending topic in a Metrology area in recent years [1]. The sound level meter, known as SLM, is considered as the most applicated device by the users among these instruments, and the customer needs related to this instrument calibration tend to increase [2]. To accommodate their necessity, the calibration of SLM has been provided by the National Standardization Agency of Indonesia (BSN) as the representative of the National Metrology Institute (NMI) of Indonesia by using the absolute method. To realize this method, it is necessary to utilize a full anechoic chamber as a calibration medium, and the laboratory standard of a microphone as a reference device [3]. Even though this method is capable to assure the calibration result so accurately, this method is difficult to be applied because of its complexity to be implemented and have a very high financial budget, especially for the secondary calibration laboratory.

Furthermore, the other method has been introduced, where it is known as the coupler method that uses a multifunction acoustic calibrator as the portable laboratory instrument, and utilize a small insulation box as a calibration medium [4]. This method is quite simpler than the previous method, and it is appropriate to be applied by the private calibration laboratories to comply with the industrial necessity for the SLM calibration that tends to increase by a year. However, this method is considered as the new method in Indonesia relatively, where the calibration result is necessary to be discussed comprehensively. According to the requirement of calibration and testing provided by ISO 17025 , the uncertainty measurement as a main part of the calibration result needs strongly to be evaluated and analyzed [5].

Therefore, the objective of this work is to provide an evaluation of the uncertainty measurement of SLM calibration by the coupler method using the statistical approximation to determine the major parameters such as standard uncertainty, combine uncertainty, degree of freedom, and expanded uncertainty in accordance with The Guide to the expressions of Uncertainty in Measurement (GUM). Moreover, a case study related to this instrument calibration is also discussed along with this paper, where the result will be compared with the acceptance limit value required by IEC 61672-1. In addition, the calibration and measurement capability (CMC) of sound level meter calibration using this method also is planned to be proposed to the Committee of National Accreditation (KAN) as the representative organization for local accreditation.

\section{MATHEMATICAL MODEL}

The calibration of sound level meter by coupler method is conducted using the multifunction acoustic calibrator as the laboratory precision instrument, and diagram of the calibration system is shown in Fig. 1.

Additionally, to optimize the calibration process, it is necessary to make use of an insulation box as the calibration 


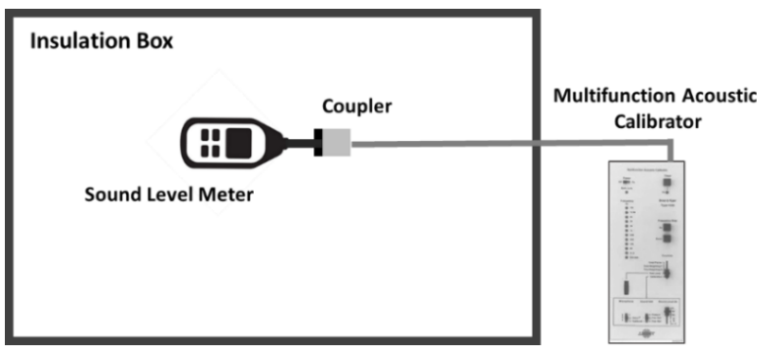

FIG. 1: Diagram of calibration system of sound level meter by coupler method

medium. In the process, sound level meter, as a unit under test (UUT), is putted into this box, and therefore, its sound pressure indication is compared directly to sound pressure level that generated by the calibrator.

Subsequently, the mathematical model of this calibration procedure can be defined, where it is stated as the deviation between the sound pressure level (L) that measured by sound level meter that later is mentioned as $\mathrm{L}_{d u t}$, and the generated $\mathrm{L}$ by the multifunction acoustic calibrator $\left(\mathrm{L}_{s t d}\right)$. In addition, these devices have a correction values as the function of frequency, where it can be obtained from the calibration result of the calibrator, meanwhile for the UUT, it is provided by the manufacturer generally. Later, these corrections are denoted as $\delta_{s t d}$ and $\delta_{d u t}$ respectively. Hence, the mathematical model for the sound level meter calibration is expressed as follow [4]:

$$
\Delta L=\left(L_{D U T}+\delta_{D U T}\right)-\left(L_{S T D}+\delta_{S T D}\right)
$$

For some cases, the information of correction values of sound level meter is not assigned by the manufacturer. Thus, it can be ignored from the Eq.(1). Subsequently, the stipulation of uncertainty budgets can be applied based on this mathematical model.

\section{UNCERTAINTY BUDGETS}

The budget of uncertainty is divided into two categories, A-type and B-type. The former is the parameter that can be obtained by applying some measurement series and generally solving with a statistical procedure, while the latter is the source that can be defined through a scientific judgment or other information that is considered to contribute to the result of measurement or calibration [6]. For the convenient, there is an additional means to simplify the determination of the uncertainty budgets beside the use of mathematical model, and therefore, it can be carried by applying the ishikawa diagram [7] that is shown in Fig.2 for the case of sound level meter calibration by coupler method.

From Fig.2, it is shown that the components of the uncertainty budgets of this calibration method can prescribe as follow:

1. According to the sound level meter as DUT, the component consists of the repeated measurement of $\mathrm{L}$ that measured by the standard instrument (repeatability), and its readability. The former is categorized as A-type of uncertainty budget. The number of the measurement data of $\mathrm{L}$ for the required frequencies is 5 times. After that, the standard deviation of the corresponding data is calculated later. Meanwhile for the later, it is classified as B-type of uncertainty budget. Further, it depends on the type of sound level meter used, where the class 1 of this handheld device has the maximum resolution up to $0.01 \mathrm{~dB}$, while for the lower class, its readability is 0.1 dB.

2. Meanwhile for the standard instrument as the next parameter that represented by multifunction acoustic calibrator, the component of the uncertainty budget comprises the calibration of a nominal sound pressure level, drift due to its use, the sound pressure response, the level of accuracy, and the total harmonic distortion with noise. The first mentioned component of this parameter is subsumed as B-type of uncertainty budget. It is obtained from the result of calibration using the laboratory standard of microphone as primary reference of acoustic measurement by insert voltage method. Next, the second components also is characterized as B-type of uncertainty budget, where it is taken from the measurement result of an intermediate check that conducted annually using the same standard and method as the previous component. Afterwards, the last three mentioned of this parameter is provided by the manufacturer through the initial certificate and manual book, and further, these components are grouped as B-type of uncertainty budget.

3. Subsequently, an additional budget also be added in this evaluation, and it is considered as a capability of the used insulation box to insulate the background noise from outside. It can be obtained by conducting measurement of L inside and outside this box separately, and later calculating deviation among these conditions. In addition, the insulation capability of this box also can be determined by performing the sound insulation $\mathrm{m}$.

\section{EVALUATION STANDARD UNCERTAINTY}

According to GUM, the evaluation method to determine a standard uncertainty of the corresponding budgets comprises A-type and B-type [6]. The former can be applied by using the statistical analysis of series of measurements. Meanwhile for the later, it is obtained by evaluation by means other than the statistical analysis of series of observations. Therefore, determination of the standard uncertainty of the aforementioned components is as follow:

1. The repeated measurement of SLM that measured by the standard instrument (repeatability). it is obtained by calculating the standard deviation after taking the data 


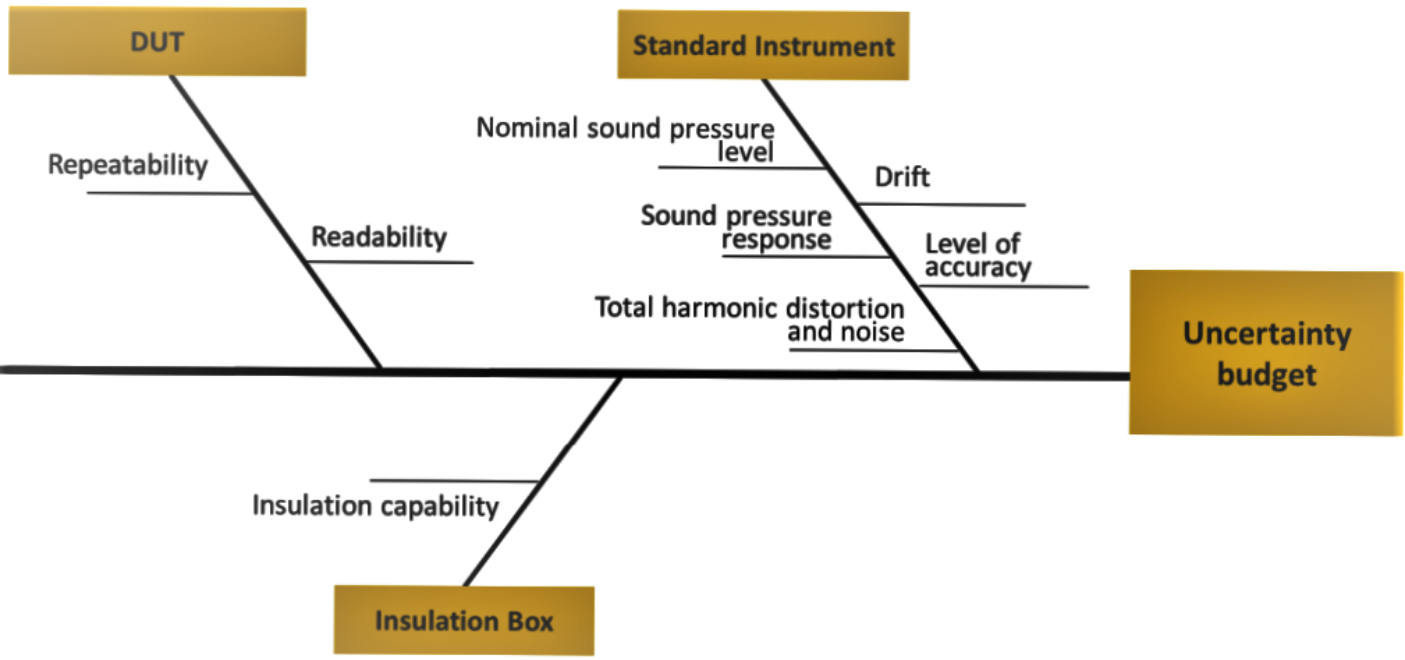

FIG. 2: Determination of uncertainty budgets by Ishikawa diagram

serially, where it can be expressed as follow:

$$
S t d e v=\sqrt{\frac{1}{N-1}} \Sigma\left(L_{i}-\bar{L}\right)
$$

where, $\mathrm{L}_{i}$ is the individual of sound pressure level measurement, while is the mean value of $\mathrm{L}$ measurements, and $\mathrm{N}$ is number of measurement series at the same conditions. After that, the standard uncertainty of this budget $\left(\mathrm{u}_{1}\right)$ can be calculated using the Eq.(3).

$$
u_{1}=\frac{S t d e v}{\sqrt{N}}
$$

2. Readability of UUT. It is determined based on its resolution, and therefore, the standard uncertainty of this component $\left(\mathrm{u}_{2}\right)$ can be assigned as follow:

$$
u_{2}=\frac{a}{\sqrt{3}}
$$

where, $a$ is a half of UUT resolution, and $\sqrt{3}$ is a divisor of the square distribution according to GUM [6]. In addition, an uncertainty budget is stated to be used this distribution if there is no information further related to the corresponding budget [6].

3. The calibration of nominal SPL of the reference instrument. It is taken from the last certificate of calibration as mentioned above, where it is stated the confidence level has a value is $95 \%$, so it should use a normal distribution that has divisor of 2, and the standard uncertainty $\left(\mathrm{u}_{3}\right)$ calculated as follow:

$$
u_{3}=\frac{U_{\text {certificate }}}{2}
$$

4. Drift of the reference instrument. As mentioned above, it is obtained from the annual check, and moreover, there is no statement and information what the used distribution and its confidence level. Hence, the appropriate distribution that should be applied of this budget is the square. Therefore, this uncertainty budget $\left(\mathrm{u}_{4}\right)$ can be calculated as follow:

$$
u_{4}=\frac{U_{d r i f t}}{\sqrt{3}}
$$

5. Sound pressure response measurement of the standard. It is found from the manual book of the corresponding instrument. By the reason of there are no information that explained the confidence level, so the square distribution is appropriate to be applied for this component. Therefore, the standard uncertainty $\left(\mathrm{u}_{5}\right)$ can be calculated as follow:

$$
u_{5}=\frac{U_{\text {responese }}}{\sqrt{3}}
$$

6. Accuracy level of the standard. The same source is obtained as the previous budget. After that, the standard uncertainty according to this component (u6) can be calculated respectively as follow::

$$
u_{6}=\frac{U_{\text {accuracy }}}{\sqrt{3}}
$$

7. Total harmonic distortion and noise $($ THD $+N)$. This is a part of calibration of nominal SPL of the reference instrument, and it can be calculated respectively as follow:

$$
u_{7}=\frac{U_{T H D}}{2}
$$

8. Capability of the insulation box to insulate the background noise from outside. The same step also applied, 
and it can be calculated as follow:

$$
u_{8}=\frac{U_{\text {insulation }}}{\sqrt{3}}
$$

In addition, the other of principal parameters also is determined, and it consists of sensitivity coefficient for the budgets of uncertainty $\left(\mathrm{c}_{i}\right)$, and a degree of freedom $\left(\mathrm{v}_{i}\right)$. The first parameter is described how the obtained measurand varies with changes in the values of the other parameters. In particular, the change in the main measurand produced by a small change of another parameter, and is given by calculating the partial derivative of the Eq.(1) to the input parameter [6]. Therefore, it can be expressed mathematically as follow:

$$
c_{i}=\frac{\partial L}{\partial x_{i}}
$$

Meanwhile for the second parameter, it depends on the type of used uncertainty method. It can be calculated by subtracting total amount of the measurement data $(\mathrm{N})$ with 1 for Atype, meanwhile for the other, it should be infinite according to JCGM and an estimation result of the published paper. Therefore, these parameters can be written serially as follow:

$$
\begin{array}{ll}
v_{i}=N-1 & \text { for type-A } \\
v_{i}=\propto & \text { for type-B }
\end{array}
$$

\section{DETERMINATION OF COMBINED STANDARD UNCERTAINTY}

Furthermore, the combined standard uncertainty can be calculated using the equation as follow [8]:

$$
u_{c}^{2}(L)=\Sigma_{i=1}^{N} c_{i}^{2} u_{i}^{2}
$$

From this equation, coefficient of sensitivity of the aforesaid budgets is calculated using the equation (10), where in this work, it is found that this value is 1 . Hence, the combined standard uncertainty is written as follow [8]:

$$
u_{c}(L)=\sqrt{u_{1}^{2}+u_{2}^{2}+u_{3}^{2}+u_{4}^{2}+u_{5}^{2}+u_{6}^{2}+u_{7}^{2}+u_{8}^{2}}
$$

\section{DETERMINATION OF EXPEANDED UNCERTAINTY}

The expanded uncertainty can be determined by multiply the combined uncertainty that has the confidence level is $67 \%$, with a coverage factor $(\mathrm{k})$. Furthermore, there are some guide to obtain $\mathrm{k}$ value, and it can be conducted by using t-student table or calculating the other parameter that is considered as effective of degree of freedom that expressed as $\mathrm{v}_{\text {eff }}$. For the first guide, the table has mentioned that the coverage factor (k) vary in the corresponding of confidence level, where it is found that this parameter has the value 1.96 for the confidence level of $95 \%$. Subsequently, the other guide also can be implemented by calculating effective degree of freedom using
Welch-Satterthwaite formula as follow [6]:

$$
v_{e f f}=\frac{u_{c}^{4}(L)}{\Sigma_{i}=1^{N} \frac{u_{i}^{4}(L)}{v_{i}}}
$$

Afterwards, $k$ can be calculated using the programmable software for the convenient, where in this work, a spreadsheet excel is used that has capability to calculate $\mathrm{k}$ using the function of TINV (probability; $\mathrm{v}_{\text {eff }}$ ), where the probability is considered as a level of hesitancy that has value of $5 \%$, and it is assigned from the normal distribution with the confidence level of $95 \%$. Finally, the expanded uncertainty (U) is determined by using the formula as follow:

$$
U(L)=k u_{c}(L)
$$

Later, this quantity will be compared with the acceptance limit value required by IEC 61672-1.

\section{CASE STUDY}

In this work, the case study of uncertainty evaluation of SLM calibration using the coupler method was provided for the A-frequency weighting parameter at the sound pressure level of $114 \mathrm{~dB}$, where the calibration process and its calculation were applied separately. The calibration was conducted in a laboratory of acoustics and vibration BSN using the system apparatus consists of a class-2 SLM as a unit under test (UUT), a reference instrument (Multifunction Acoustic Calibrator $B \& K 4226$ ), and an insulation box.

During the calibration, there was no alteration of environmental conditions, where it was recorded as $24.2^{\circ} \mathrm{C}, 63 \% \mathrm{RH}$, and $100.0 \mathrm{kPa}$ for ambient conditions of temperature, relative humidity, and air pressure respectively inside the insulation box. The data was taken five times for the corresponding frequencies, where the details of the result shown in Table I.

The determination of uncertainty measurement for SLM calibration by the coupler method is written in Table I. For the first parameter represented by the UUT, the uncertainty component consists of the repeated measurement and its readability. The former has the standard uncertainty values vary for the measurement frequencies, where the maximum value is found at the frequency of $63 \mathrm{~Hz}, 125 \mathrm{~Hz}, 1 \mathrm{kHz}, 2 \mathrm{kHz}$, and $8 \mathrm{kHz}$ that its value is about $0.02 \mathrm{~dB}$. Meanwhile for the later, the standard uncertainty value is seen to be equal for the corresponding frequencies that are obtained and calculated using B-type.

Subsequently, the second parameter represented by the Multifunction Acoustic Calibrator provides the most components that contribute to the uncertainty calculation, and it can be seen that the standard uncertainty tends to be steady for the component that is obtained from SPL calibration and THD $+\mathrm{N}$ report. Subsequently, the remain components that comprise the drift value of this standard instrument therewith sound pressure response and level of accuracy are shown that the standard uncertainty values lean to go up and down beyond these frequencies. Afterward, the last parameter is given by 


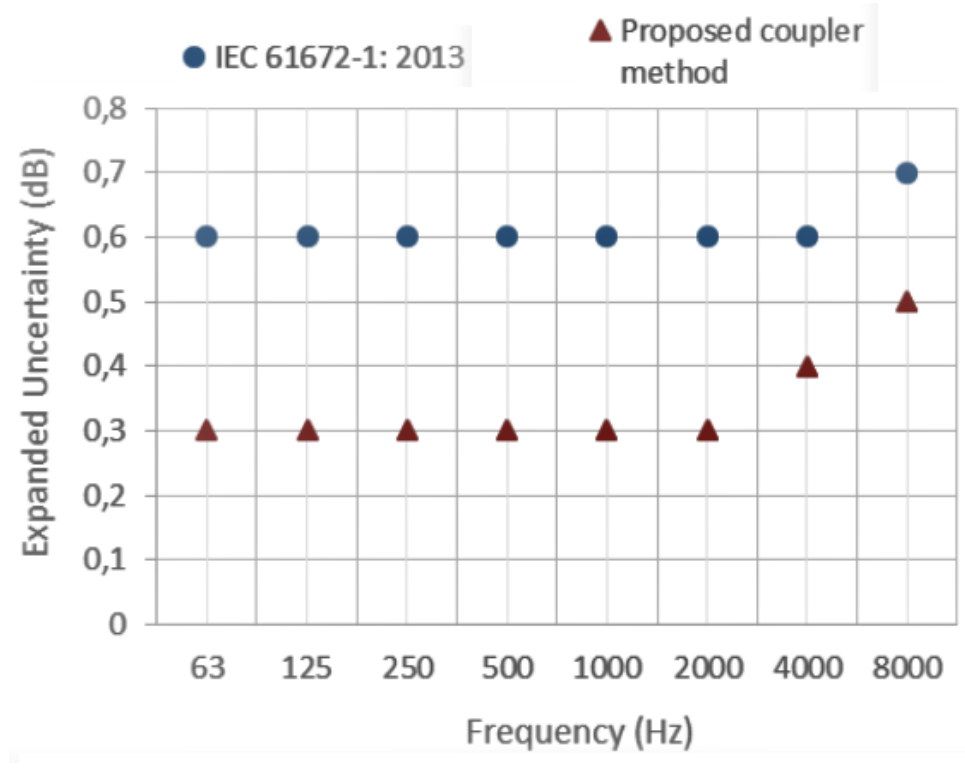

FIG. 3: Expanded uncertainties comparison (Blue: IEC 61672-1:2013, Red: Proposed coupler method)

TABLE I: Calculation of uncertainty measurement of sound level meter calibration for SPL $114 \mathrm{~dB}$.

\begin{tabular}{|c|c|c|c|c|c|c|c|c|c|c|}
\hline \multirow[t]{2}{*}{ No } & \multicolumn{3}{|c|}{ Uncertainty budged } & \multicolumn{7}{|c|}{ Standard Uncertainty (dB) } \\
\hline & Parameter & Component & $63 \mathrm{~Hz}$ & $125 \mathrm{~Hz}$ & $250 \mathrm{~Hz}$ & $500 \mathrm{~Hz}$ & $1 \mathrm{kHz}$ & $2 \mathrm{kHz}$ & $4 \mathrm{kHz}$ & $8 \mathrm{kHz}$ \\
\hline \multirow[t]{2}{*}{1} & Sound Level & Repeatability (Tipe A) & 0.02 & 0.02 & 0.00 & 0.00 & 0.02 & 0.02 & 0.00 & 0.02 \\
\hline & Meter UUT & Readability & 0.03 & 0.03 & 0.03 & 0.03 & 0.03 & 0.03 & 0.03 & 0.03 \\
\hline \multirow[t]{5}{*}{2} & Multifunction & SPL of $114 \mathrm{~dB}$ & 0.06 & 0.06 & 0.05 & 0.05 & 0.05 & 0.06 & 0.06 & 0.05 \\
\hline & Calibrator & Drift & 0.04 & 0.04 & 0.04 & 0.01 & 0.04 & 0.03 & 0.02 & 0.02 \\
\hline & B\&K 24226 & Sound pressure response & 0.07 & 0.06 & 0.06 & 0.06 & 0.06 & 0.08 & 0.11 & 0.17 \\
\hline & & Level of accuracy & 0.09 & 0.09 & 0.06 & 0.06 & 0.09 & 0.09 & 0.09 & 0.14 \\
\hline & & $\mathrm{THD}+\mathrm{N}$ & 0.00 & 0.00 & 0.00 & 0.00 & 0.00 & 0.00 & 0.00 & 0.00 \\
\hline \multirow[t]{5}{*}{3} & Insulation box & Insulation capacity & 0.06 & 0.06 & 0.06 & 0.06 & 0.06 & 0.06 & 0.06 & 0.06 \\
\hline & & $\mathrm{uc}(\mathrm{dB})$ & 0.15 & 0.14 & 0.13 & 0.12 & 0.14 & 0.15 & 0.17 & 0.24 \\
\hline & & $\mathrm{v}_{e f f}$ & 207.30 & 202.41 & 255.97 & 218.66 & 203.73 & 200.68 & 167.85 & 124.69 \\
\hline & & $\mathrm{k} 95 \%$ & 1.97 & 1.97 & 1.97 & 1.97 & 1.97 & 1.97 & 1.97 & 1.98 \\
\hline & & $\mathrm{u}(\mathrm{dB})$ & 0.29 & 0.28 & 0.25 & 0.23 & 0.28 & 0.29 & 0.33 & 0.48 \\
\hline
\end{tabular}

the insulation box that assists the standard uncertainties is about $0.06 \mathrm{~dB}$ for all frequencies.

After that, the combined uncertainty $\left(\mathrm{u}_{c}\right)$ and the effective of degree of freedom $\left(\mathrm{v}_{e f f}\right)$ is calculated, and therefore, the multifunction acoustic calibrator withal the sound pressure response and level of accuracy has the highest contribution to calculate the combined uncertainty at the frequency of 8000 $\mathrm{Hz}$ that its value is $0.24 \mathrm{~dB}$. After that, by determining $\mathrm{k}$ value for the confidence level of $95 \%$, the expanded uncertainty $\left(\mathrm{U}_{c}\right)$ can be obtained, and its value reaches up to $0.48 \mathrm{~dB}$ of this frequency. Meanwhile for the other frequencies, it has a value at the range of $0.24-0.33 \mathrm{~dB}$.

In contrast to using the absolute method that has the expanded uncertainty tend to be flat in this frequency range, the coupler method of SLM calibration tends to increase at the high frequencies. It can be explained that at this frequency, the sound that generates by the standard instrument is easier to be flanking transmission when the microphone of SLM is coupled to this standard. It can be seen in the datasheet of multifunction acoustic calibrator that shown that the level of accuracy and SPL response has the biggest correction value at the high frequencies.

In addition, comparison of the determined expanded uncertainties and its acceptance values provided by IEC 61672-1: 2013 [9] is shown in Fig.3. The obtained expanded uncertainty values in this work that represented by the red triangles are below to the maximum of acceptance values that expressed by the blue dots for all frequencies as seen in Fig.3. The two curves have a difference of its trend slightly, where it is shown that the obtained Uc start to increase about $0.1 \mathrm{~dB}$ at frequency of $4000 \mathrm{~Hz}$ and go up to $8000 \mathrm{~Hz}$ with the same increasing value. Meanwhile for the curve that given by IEC 61672-1: 2013 , it is equal from the initial frequency to $4000 \mathrm{~Hz}$, after that, the similar condition is found as well as the previous curve. Hence, the results of this work are still acceptable and reasonable to be applicated for the calibration of sound level 
meter further. In addition, the expanded uncertainty is estimated to has a lower value when using the sound level meter test that has better resolution, and it has alteration about 0.1 $\mathrm{dB}$ for whole frequencies.

\section{CONCLUSION}

The evaluation of uncertainty measurement of sound level meter calibration by coupler method has been conducted by Research Group for Acoustics and Vibration at laboratory acoustics and vibration-BSN. Besides, the determination of an uncertainty budget, and other important attributes also have been provided. The multifunction acoustic calibrator along with the sound pressure response and level of accuracy has the highest contribution to determine the expanded uncertainty at the high frequency. This result also has shown that the ob- tained expanded uncertainty values in this work are acceptable, and therefore, it is reasonable to be proposed to the Committee of National Accreditation (KAN) as the representative organization for local accreditation. In addition, this result is strongly recommended to be compared with another method to evaluate uncertainty measurement such as the Monte-Carlo method.

\section{Acknowledgments}

The authors acknowledge the financial support from Ministry of Research and Technology/National Agency for Research and Innovation of the Republic of Indonesia through the scheme of Incentive Research Program for the National Innovation System in 2020/2021.
[1] C. Series, "Measurement traceability of acoustics and vibration instruments in Indonesia Measurement traceability of acoustics and vibration instruments in Indonesia", 2018.

[2] D. Hermawanto et al., "Development of Decibel Stepped Attenuator for Automated Sound Level Meter Calibration", Mapan J. Metrol. Soc. India, vol. 35, no. 1, pp. 8186, 2020.

[3] N. Garg, et al., "Measurement Uncertainty in Microphone FreeField Comparison Calibrations", MAPAN, 2019.

[4] I.MA.2.06, "Calibration Procedure for Sound Level Meter by Using Multi Frequency Acoustic Calibrator", 2015.

[5] SNI ISO/IEC 17025:2017 "Persyaratan umum kompetensi laboratorium pengujian dan kalibrasi", 2019.
[6] JCGM, "Evaluation of measurement data Guide to the expression of uncertainty in measurement", no. September, 2008.

[7] D. A. Giardino and J. P. Seiler, "Uncertainties associated with noise dosimeters in mining", vol. 100, no. May 1996, pp. 15711576, 2014.

[8] I.MA.2.06.U-E.2-R.0, "Evaluasi Ketidakpastian Kalibrasi Sound Level Meter Menggunakan Multifunction Acoustic Calibrator", 2019.

[9] IEC 61672-1, "Electroacoustics-Sound Level Meters-Part 1: Spesification”, International Electroacoustics Commision, 2013. 